\title{
Erratum to: Controlled Natural Language
}

\author{
Brian Davis ${ }^{1(\bowtie)}$, Gordon J. Pace ${ }^{2}$, and Adam Wyner ${ }^{3}$ \\ 1 National University of Ireland, Galway, Ireland \\ brian.davis@insight-centre.org \\ ${ }^{2}$ University of Malta, Msida, Malta \\ ${ }^{3}$ University of Aberdeen, Aberdeen, UK
}

\section{Erratum to:}

\section{B. Davis et al. (Eds.)}

\section{Controlled Natural Language}

DOI: 10.1007/978-3-319-41498-0

The original version of the preface was revised:

The name of an invited speaker as well as several typos were corrected. 